\title{
Association of ABC Transporter With Resistance to FK866, a NAMPT Inhibitor, in Human Colorectal Cancer Cells
}

\author{
YOKO OGINO $^{1,2}$, AKIRA SATO ${ }^{1}$, YOHEI KAWANO ${ }^{3}$, TAKAO AOYAMA ${ }^{3}$, \\ FUMIAKI UCHIUMI ${ }^{2}$ and SEI-ICHI TANUMA ${ }^{4}$
}

\begin{abstract}
${ }^{1}$ Department of Biochemistry, Faculty of Pharmaceutical Sciences, Tokyo University of Science, Noda, Japan; ${ }^{2}$ Department of Gene Regulation, Faculty of Pharmaceutical Sciences, Tokyo University of Science, Noda, Japan; ${ }^{3}$ Department of Pharmacotherapeutics, Faculty of Pharmaceutical Sciences, Tokyo University of Science, Noda, Japan;

${ }^{4}$ Department of Genomic Medicinal Science, Research Institute for Science and Technology, Organization for Research Advancement, Tokyo University of Science, Noda, Japan
\end{abstract}

\begin{abstract}
Background/Aim: Nicotinamide phosphoribosyltransferase (NAMPT), a key enzyme in the $N A D^{+}$biosynthetic pathway, is a drug target of potent anticancer candidates, including FK866 and other reported NAMPT inhibitors. However, it is known that NAMPT point-mutations render resistance to specific NAMPT inhibitors in several cancer cells. We investigated the resistance mechanisms of NAMPT inhibitor FK866 in human colorectal cancer (CRC) cells. Materials and Methods: We used CRC human cell line HCT116 to determine the expression profiles of FK866-sensitive parental HCT116 cells and FK866-resistant HCT116 (HCT116R ${ }^{\text {FK866) }}$ cells by DNA microarray analysis. The levels of multidrug resistance protein 1 (MDRI) were assessed via western blot. In addition, we analyzed the sensitivity of FK866 in parental HCT116 cells and HCT116R ${ }^{\text {FK866 }}$ cells by co-treatment with MDR1 inhibitor verapamil. Results: Our results revealed an association between ATP-binding cassette $(A B C)$ transporter gene $A B C B 1$ and resistance to NAMPT inhibitor FK866 in both HCT116R ${ }^{F K 866}$ cells and parental HCT116 cells. The expression of ABCB1, which encodes MDR1, was lower in HCT116R FK866 cells than in parental HCT116 cells. Furthermore, the protein level of MDR1/ATP-binding cassette sub-family B member 1 (ABCB1) was 0.5-fold lower in HCT116R FK866 cells than in parental HCT116 cells. Additionally, HCT116R FK866 cells showed improved sensitivity to FK866 when co-treated with verapamil,
\end{abstract}

This article is freely accessible online.

Correspondence to: Akira Sato, Department of Biochemistry, Faculty of Pharmaceutical Sciences, Tokyo University of Science, 2641 Yamazaki, Noda, Chiba 278-8510, Japan. Tel/Fax: +81 471213620, e-mail: akirasat@rs.tus.ac.jp

Key Words: ABC transporter, drug resistance, FK866, NAMPT, NAMPT inhibitor, verapamil. an ABCB1 inhibitor. Interestingly, the efficacy of FK866 in parental HCT116 cells was the same for the treatment with FK866 alone or in combination with verapamil. Conclusion: The change in expression of $A B C B 1$ plays a key role in $C R C$ drug resistance to NAMPT inhibitor FK866. This suggests that the $M D R 1 / A B C B 1$ mechanism may regulate the resistance of anticancer NAMPT inhibitor FK866.

Nicotinamide phosphoribosyltransferase (NAMPT) is a ratelimiting enzyme in the salvage pathway of nicotinamide (NAM) in nicotinamide adenine dinucleotide $\left(\mathrm{NAD}^{+}\right)$ synthesis (1-4). NAMPT is a potential anticancer drug target, and many drug candidates have been developed that inhibit its enzymatic activity $(1,5)$, including FK866 (also known as APO866 and WK175) $(6,7), \mathrm{CHS}-828$ (also known as GMX1778) (8-11), GNE-617 (12), and STF118804 (13). Acquired resistance to anticancer NAMPT inhibitors appears to be due to alterations in NAMPT itself (14-16). There have been previous reports that NAMPT point-mutations confer resistance to specific NAMPT inhibitors (14-16). Recently, we produced FK866-resistant HCT116R ${ }^{\text {FK866 }}$ cells from human colorectal cancer (CRC) HCT116 cells $(17,18)$. Whole-exome sequencing of the NAMPT gene revealed two single point-mutations, H191R and $\mathrm{K} 342 \mathrm{R}$, in NAMPT in HCT116R ${ }^{\mathrm{FK} 866}$ cells, of which only K342R was present in the parental HCT116 cells (17, 18). Additionally, we used a NAMPT-immunoprecipitated proteomics approach to demonstrate that NAMPT in HCT116 cells, but not HCT116 ${ }^{\text {FK866 }}$ cells, interacted with the truncated POTE (expressed in prostate, ovary, testis, and placenta) ankyrin domain family member E (tPOTEE) and $\beta$-actin. Moreover, we reported that the NAMPT H191R variant in HCT116 $\mathrm{R}^{\mathrm{FK} 866}$ cells might prevent interaction with the two identified binding partners (tPOTEE and $\beta$-actin), resulting in cellular resistance to diverse NAMPT inhibitors (17). We also demonstrated that HCT116R ${ }^{\text {FK866 }}$ 
A<smiles>O=C(/C=C/c1cccnc1)NCCCCC1CCN(C(=O)c2ccccc2)CC1</smiles>

FK866
B<smiles>COc1ccc(CCN(C)CCCC(C#N)(c2ccc(OC)c(OC)c2)C(C)C)cc1OC</smiles>

Verapamil

Figure 1. Chemical structures of NAMPT inhibitor FK866 (A) and ABC transporter inhibitor verapamil (B).

cells were more sensitive to the anticancer 5-fluorouracil and cisplatin and $\gamma$-ray irradiation compared with parental HCT116 cells (18).

Cancer drug resistance is a challenge that needs to be overcome to achieve effective anticancer chemotherapy. However, the underlying mechanisms can differ depending on the medicine, and common cancer resistance mechanisms include drug inactivation, drug efflux, drug-targeted alterations, bypass pathway activation, DNA damage repair, and cell death escape (19). Importantly, multidrug resistance (MDR) in cancer cells can significantly attenuate the response to chemotherapy (20-22). The major mechanism involved in conferring MDR is the overexpression of ATPbinding cassette $(\mathrm{ABC})$ transporters, which can increase the efflux of drugs from cancer cells, thereby decreasing intracellular drug concentration (20-22).

The relationship between the mechanism of NAMPT resistance and $\mathrm{ABC}$ transporter is unelucidated. To this end, we investigated the association of $\mathrm{ABC}$ transporter with resistance to FK866, a NAMPT inhibitor, in human CRC HCT116 cells.

\section{Materials and Methods}

Reagents. The anticancer NAMPT inhibitor $\mathrm{FK} 866 \mathrm{HCl}$ was obtained from Focus Biomolecules (Plymouth Meeting, PA, USA) and was stored as a $10 \mathrm{mM}$ stock in ultra-pure water at $-20^{\circ} \mathrm{C}$. The $\mathrm{ABC}$ transporter inhibitor verapamil was obtained from FUJIFILM Wako Pure Chemical Corp. (Doshomachi, Osaka, Japan) and was stored as a $20 \mathrm{mM}$ stock in DMSO at $-20^{\circ} \mathrm{C}$.

Cell culture. The human CRC cell line HCT116 was obtained from the American Type Culture Collection (Manassas, VA, USA). FK866resistant HCT116 (HCT116R ${ }^{\text {FK866) }}$ cells were developed according to the previously described method (17). Parental HCT116 and HCT116R FK866 cell lines were cultured in Dulbecco's modified Eagle's medium containing $10 \%$ heat-inactivated fetal bovine serum, $100 \mathrm{U} / \mathrm{ml}$ penicillin, and $100 \mu \mathrm{g} / \mathrm{ml}$ streptomycin in an incubator a $37^{\circ} \mathrm{C}$ in an atmosphere of $5 \% \mathrm{CO}_{2}$ at $100 \%$ relative humidity.
Clonogenicity assay. Clonogenicity assays were performed as previously described $(17,18)$. Briefly, parental HCT116 and

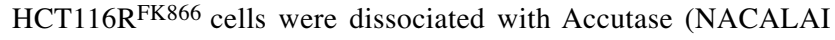
TESQUE INC., Nijo Karasuma, Kyoto, Japan), suspended in medium, inoculated into six-well plates (200 cells/well) in triplicate, and then incubated overnight. The cells were treated with various concentrations of drugs (FK866 only or co-treatment with verapamil), a concentration of DMSO, or ultra-pure water (control). After 10 days' incubation, the cells were fixed with a solution of $4 \%$ formaldehyde-phosphate-buffered saline and stained with $0.1 \%(\mathrm{w} / \mathrm{v})$ crystal violet. The number of colonies per well were then counted.

Microarray analysis. RNA extraction was performed as described previously $(23,24)$. Briefly, total RNA was extracted using QIAshredder spin columns and an RNeasy Mini Kit (both QIAGEN, Hilden, Germany) as per the manufacturer's instructions. DNA microarray analysis of the parental HCT116 and HCT116R FK866 cells was performed by TAKARA Bio Inc. (Shiga, Japan) using a SurePrint G3 Human Gene Expression $8 \times 60 \mathrm{~K}$ v3 Microarray (Agilent Technologies, Santa Clara, CA, USA) to determine the expression profiles of the cells.

Western blot analysis. Western blot analysis was performed as described previously $(17,25)$. The following antibodies were used: rabbit anti-MDR1 (1:1,000; Abcam, Cambridge, UK), rabbit antiglyceraldehyde 3-phosphate dehydrogenase (GAPDH; 1:20,000; Trevigen, Gaithersburg, MD, USA), and horseradish peroxidaselabelled anti-rabbit immunoglobulin (Ig)G (1:20,000; GE Healthcare, Pittsburgh, PA, USA).

Statistical analysis. The data were presented as the means \pm standard deviation (SD). The significance of differences among groups was evaluated using Student's $t$-test; $p<0.05$ was considered statistically significant.

\section{Results}

To investigate the resistance mechanisms of NAMPT inhibitor FK866 (Figure 1A) in human CRC, we analyzed the comprehensive gene expression profiles of FK866-resistant HCT116R ${ }^{\text {FK866 }}$ cells and FK866-sensitive parental HCT116 
Table I. Gene expression of ABCB1 in parental HCT116 and HCT116RFK866 cells as determined by microarray analysis.

\begin{tabular}{lccccc}
\hline Gene symbol & Gene description & S (signal) & R (signal) & FC (R/S) & $p$-Value \\
\hline$A B C B 1$ & ATP-binding cassette, sub-family B (MDR/TAP), member 1 & 153.0 & 73.5 & 0.48 & 0.004 \\
\hline
\end{tabular}

S, FK866-sensitive parental HCT116 cells; R, FK866-resistant HCT116R FK866 cells; FC, fold change (HCT116R FK866 cells vs. HCT116 cells). The signal values represent the average signal intensity for the microarray probe (probe ID: A_23_P82523) from three independent experiments.

Table II. Gene mutation status of multidrug resistance gene ABCB1 in HCT116 and HCT116R FK866 cells.

\begin{tabular}{|c|c|c|}
\hline \multirow[t]{2}{*}{ Gene symbol } & \multicolumn{2}{|c|}{ Gene mutations } \\
\hline & HCT116 & HCT116R FK866 \\
\hline$A B C B 1$ & $\begin{array}{l}m t(\text { Ile1145Ile)het } \\
m t(\text { Ser893Ala)het } \\
m t(\text { Asp603His)het } \\
m t \text { (Gly412Gly)het } \\
m t \text { (Asn21Asp)het }\end{array}$ & $\begin{array}{l}m t(\mathrm{Ile} 1145 \mathrm{Ile}) h e t \\
m t(\mathrm{Ser} 893 \mathrm{Ala}) h e t \\
m t(\mathrm{Asp} 603 \mathrm{His}) h e t \\
m t(\mathrm{Gly} 412 \mathrm{Gly}) h e t \\
m t \text { (Asn21Asp)het }\end{array}$ \\
\hline
\end{tabular}

$m t$, Mutation-type; het, heterozygous.

cells using DNA microarray technology (data not shown). In the microarray analysis, the expression of multidrug resistancerelated $A B C B 1$ was decreased in HCT116R $\mathrm{R}^{\text {FK866 cells }}$ compared with parental HCT116 cells (Table I). Previously, we used whole-exome sequencing analysis to demonstrate that the gene mutation status of $A B C B 1$ was similar in $\mathrm{HCT} 116 \mathrm{R}^{\mathrm{FK} 866}$ and parental HCT116 cells (Table II) (18). However, the relationship between the mechanism of NAMPT inhibitor FK866 resistance and $\mathrm{ABC}$ transporter remained unelucidated. Therefore, we investigated the association of $\mathrm{ABC}$ transporter and FK866 resistance in our established human CRC FK866resistant HCT116 ${ }^{\mathrm{FK} 866}$ (17) and FK866-sensitive parental HCT116 cells. ABCB1/MDR1 is a glycosylated 170-kDa transmembrane protein that is encoded by the $A B C B 1$ gene, and the drug efflux pump of the family of $\mathrm{ABC}$ transporters is well known (20-22). Microarray analysis revealed that the gene expression of $A B C B 1$ was 0.5 -fold lower in $\mathrm{HCT} 116 \mathrm{R}^{\mathrm{FK} 866}$ cells than in parental HCT116 cells (Table I). For western blot validation, the protein level of $A B C B 1$ was 0.5 -fold lower in HCT116R ${ }^{\text {FK866 }}$ cells than in parental HCT116 cells (Figure 2). These findings suggested that MDR1/ABCB1 was expressed at lower levels in FK866-resistant HCT116R ${ }^{\text {FK866 }}$ cells compared to parental HCT116 cells.

To explore how the multidrug-resistant $A B C B 1$ function conferred resistance to NAMPT inhibitor FK866, we analyzed the sensitivity of FK866 in HCT116R ${ }^{\text {FK866 }}$ cells and parental HCT116 cells by co-treatment with verapamil (Figure 1B), an $\mathrm{ABC}$ transporter $\mathrm{ABCB} 1$ inhibitor $(20,21)$.

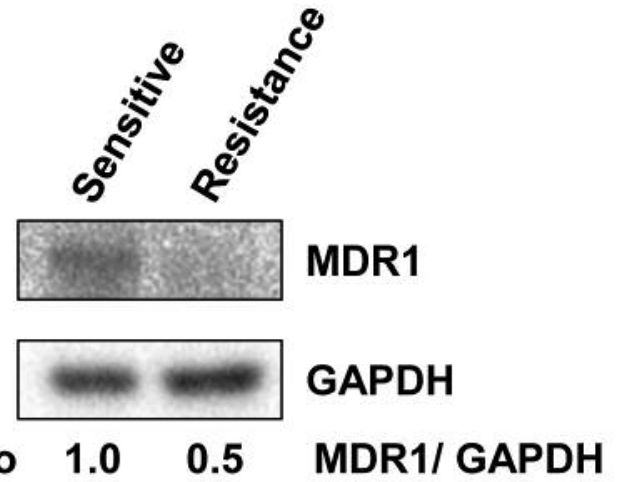

Figure 2. Protein levels of MDR1 in FK866-resistant HCT116R FK866 and parental HCT116 human CRC cells. Whole-cell lysates were prepared from parental HCT116 and HCT116RFK866 cells. Protein levels of MDRI and GAPDH were examined by western blot analysis. The expression of GAPDH was used as an internal control. Data are representative of at least three independent experiments. Levels of $M D R 1$ protein are represented by the ratio of MDR1 density to GAPDH density relative to the value for parental HCT116 cells. Results represent the averages of three independent experiments.

Interestingly, HCT116R $\mathrm{RK}^{\mathrm{F} 866}$ cells showed improved sensitivity to FK866 when co-treated with verapamil. In contrast, the sensitivity of FK866 in parental HCT116 cells was unaffected by the verapamil co-treatment (Figure 3 ). The $50 \%$ effective concentration $\left(\mathrm{EC}_{50}\right)$ of $\mathrm{FK} 866$ in parental HCT116 and HCT116R ${ }^{\text {FK866 }}$ cells with or without verapamil co-treatment was determined by colony formation assay. The $\mathrm{EC}_{50}$ of FK866 in HCT116R ${ }^{\mathrm{FK} 866}$ cells was higher with FK866 alone $\left(\mathrm{EC}_{50}=6,650 \mathrm{nM}\right.$ in FK866) compared with cotreatment with verapamil $\left(\mathrm{EC}_{50}=2,700 \mathrm{nM}\right.$ in $\left.\mathrm{FK} 866\right)$. In contrast, the $\mathrm{EC}_{50}$ of FK866 in parental HCT116 cells was lower with FK866 alone $\left(\mathrm{EC}_{50}=11 \mathrm{nM}\right.$ in FK866) compared to co-treatment with verapamil $\left(\mathrm{EC}_{50}=15 \mathrm{nM}\right.$ in FK866) (Figure 3A and Table III). Regarding the sensitivity index of FK866, HCT116R ${ }^{\text {FK866 }}$ cells were 2.5-fold more sensitive to FK866 when co-treated with verapamil. In contrast, HCT116 cells were almost similar in sensitivity to FK866 with verapamil co-treatment. These findings suggested that $A B C B 1$ machinery regulates NAMPT inhibitor FK866 resistance in HCT116R $\mathrm{R}^{\mathrm{FK} 866}$ cells. 
A

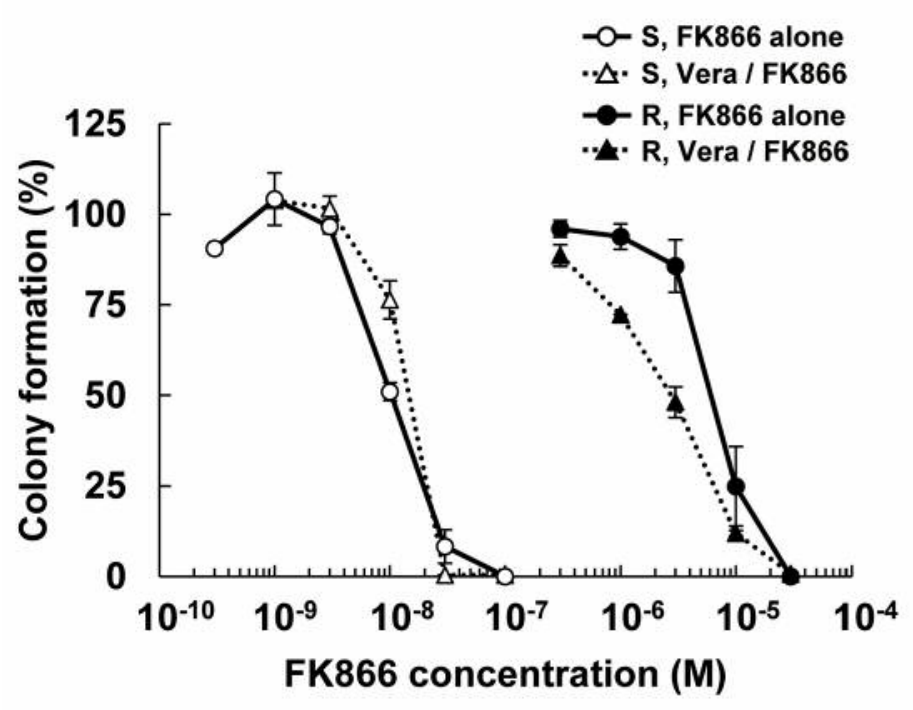

B

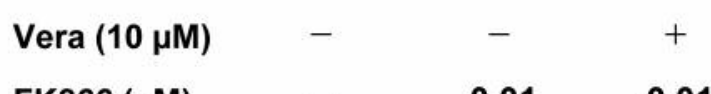

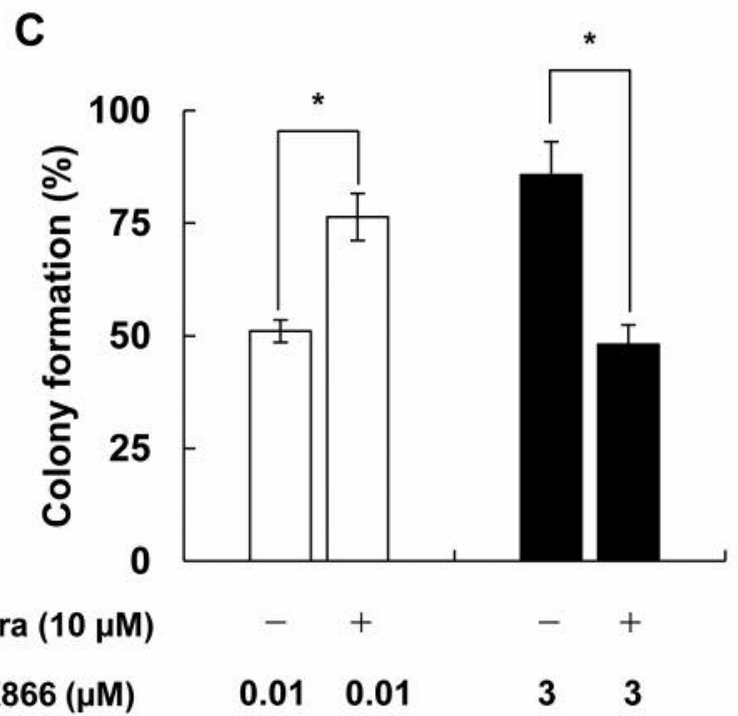

\section{Discussion}

NAMPT, a key enzyme in the $\mathrm{NAD}^{+}$biosynthetic pathway, is a molecular target of potent anticancer candidates, including FK866 and other reported NAMPT inhibitors (1-4). However, many studies have previously reported that NAMPT pointmutations render resistance to specific NAMPT inhibitors in several cancer cell types (14-16). We investigated the resistance mechanisms of NAMPT inhibitor FK866 in human CRC HCT116 cells $(17,18)$. Our findings revealed that multidrug resistant-related $A B C B 1$ (the gene encoding MDR1) is expressed at a lower level in FK866-resistant HCT116R ${ }^{\mathrm{FK} 866}$
Figure 3. The sensitivity to FK866 in FK866-resistant HCT116RFK866 and parental HCT116 human CRC cells with verapamil co-treatment. (A) Colony formation by parental HCT116 and HCT116R FK866 cells after 10 days' treatment with FK866 alone or co-treatment with verapamil. Results are the average of two independent experiments. Error bars show the \pm standard error (SE) in triplicate experiments. (B) Drug sensitivities of HCT116 and HCT116RFK866 in the colony formation assay. HCT116 cells were treated with $10 \mathrm{nM} \mathrm{FK866}$ alone or $10 \mu M$ verapamil plus $10 \mathrm{nM} \mathrm{FK866.} \mathrm{HCT116RFK866} \mathrm{cells} \mathrm{were}$ treated with 3,000 nM FK866 alone or $10 \mu \mathrm{M}$ verapamil plus 3,000 nM FK866. (C) Colony formation (\%) is the average of two independent experiments. Error bars show \pm SE of triplicate experiments. Values are standardized by a no-treatment control (no drug, solvent alone) in parental HCT116 and HCT116RFK866 cells, respectively. An asterisk indicates a statistically significant differences (Student's t-test, $p<0.01$ ).

cells compared to parental HCT116 cells (Figure 2 and Table I). We previously demonstrated that FK866-resistant HCT116 $\mathrm{R}^{\mathrm{FK} 866}$ cells, which are resistant to other classes of NAMPT inhibitors (e.g., CHS-828, GNE-617, and STF118804), were more sensitive to the anticancer medicines 5-fluorouracil and cisplatin compared to parental HCT116 cells (18). Interestingly, $\mathrm{HCT} 116 \mathrm{R}^{\mathrm{FK} 866}$ cells showed a 2.5 -fold higher sensitivity (i.e., less resistance) to FK866 with verapamil cotreatment. This suggests that ABCB1 may be involved in the efflux of NAMPT inhibitor FK866 in HCT116R ${ }^{\mathrm{FK} 866}$ cells. We consider that FK866-resistant HCT116R ${ }^{\mathrm{FK} 866}$ cells become resistant to NAMPT inhibitor FK866 by down-regulating 
Table III. Sensitivity to FK866 in parental HCT116 and HCT116R FK866 cells with co-treatment of verapamil.

\begin{tabular}{lccc}
\hline Cell line & \multicolumn{2}{c}{ FK866 $\left(\mathrm{EC}_{50}, \mathrm{nM}\right)$} & \\
\cline { 2 - 3 } & Verapamil (-) & Verapamil (+) & Sensitivity index \\
\hline HCT116 & 11 & 15 & 0.7 \\
HCT116R FK866 & 6,650 & 2,700 & 2.5 \\
\hline
\end{tabular}

The cells were treated as described in the legend to Figure 3 . The $\mathrm{EC}_{50}$ values are the average of triplicate determinations obtained from two independent experiments. The sensitivity index was determined as the $\mathrm{EC}_{50}$ values of FK866 alone divided by the $\mathrm{EC}_{50}$ values of $\mathrm{FK} 866$ in combination with verapamil and FK866 in HCT116 and HCT116R FK866 cells, respectively.

$A B C B 1$ expression. Importantly, the sensitivity of FK866 in parental HCT116 cells was unaffected by verapamil cotreatment. Interestingly, many previous reports demonstrated that the overexpression of $A B C B 1$ is responsible for the resistance against anticancer drugs, e.g., cisplatin, etoposide, paclitaxel, and doxorubicin, in several cancer cells (20, 26-28). Conversely, our finding suggests that the lower expression of $A B C B 1$ is associate with anticancer FK866 resistance in the FK866-resistant HCT116R ${ }^{\text {FK866 }}$ cells. These results indicated the difference in the ABCB1 function between HCT116R ${ }^{\text {FK866 }}$ cells and parental HCT116 cells. We propose that this difference was regulated by post-transcriptional modification (e.g., protein modification by phosphorylation, the interaction of long noncoding RNAs, or other factors). We are currently investigating the association of $\mathrm{ABC}$ transporter $\mathrm{ABCB} 1$ and other family members with NAMPT inhibitor FK866 resistance mechanisms in human CRC HCT116 cells. Our data indicate that FK866resistance in human CRC cells is responsible for the diverse mechanisms; NAMPT mutation, differences in the components of NAMPT complex, and different drug efflux machinery. Finally, these novel findings provide a better understanding of the resistance mechanisms of the anticancer NAMPT inhibitors.

\section{Conflicts of Interest}

None.

\section{Authors' Contributions}

AS: Conceived and designed the experiments. YO and AS: Performed the experiments. YO, AS, YK, TA, FU and ST: Analyzed the data. YO and AS: Wrote the paper.

\section{Acknowledgements}

This work was supported by a Research Education Fund for Tokyo University of Science. We would also like to thank Enago (www.enago.jp) for English language editing.

\section{References}

1 Galli U, Travelli C, Massarotti A, Fakhfouri G, Rahimian R, Tron GC and Genazzani AA: Medicinal chemistry of nicotinamide phosphoribosyltransferase (nampt) inhibitors. J Med Chem 56(16): 6279-6296, 2013. PMID: 23679915. DOI: $10.1021 / \mathrm{jm} 4001049$

2 Sampath D, Zabka TS, Misner DL, O'Brien T and Dragovich PS: Inhibition of nicotinamide phosphoribosyltransferase (nampt) as a therapeutic strategy in cancer. Pharmacol Ther 151: 16-31, 2015. PMID: 25709099. DOI: 10.1016/j.pharmthera. 2015.02.004

3 Kennedy BE, Sharif T, Martell E, Dai C, Kim Y, Lee PW and Gujar SA: Nad(+) salvage pathway in cancer metabolism and therapy. Pharmacol Res 114: 274-283, 2016. PMID: 27816507. DOI: $10.1016 /$ j.phrs.2016.10.027

4 Tanuma S, Sato A, Oyama T, Yoshimori A, Abe H and Uchiumi F: New insights into the roles of nad+-poly(adp-ribose) metabolism and poly(adp-ribose) glycohydrolase. Curr Protein Pept Sci 17(7): 668-682, 2016. PMID: 27817743.

5 Roulston A and Shore GC: New strategies to maximize therapeutic opportunities for nampt inhibitors in oncology. Mol Cell Oncol 3(1): e1052180, 2016. PMID: 27308565. DOI: $10.1080 / 23723556.2015 .1052180$

6 Hasmann $M$ and Schemainda I: Fk866, a highly specific noncompetitive inhibitor of nicotinamide phosphoribosyltransferase, represents a novel mechanism for induction of tumor cell apoptosis. Cancer Res 63(21): 7436-7442, 2003. PMID: 14612543.

7 Holen K, Saltz LB, Hollywood E, Burk K and Hanauske AR: The pharmacokinetics, toxicities, and biologic effects of fk866, a nicotinamide adenine dinucleotide biosynthesis inhibitor. Invest New Drugs 26(1): 45-51, 2008. PMID: 17924057. DOI: $10.1007 / \mathrm{s} 10637-007-9083-2$

8 Hjarnaa PJ, Jonsson E, Latini S, Dhar S, Larsson R, Bramm E, Skov $\mathrm{T}$ and Binderup L: Chs 828, a novel pyridyl cyanoguanidine with potent antitumor activity in vitro and in vivo. Cancer Res 59(22): 5751-5757, 1999. PMID: 10582695.

9 Hovstadius P, Larsson R, Jonsson E, Skov T, Kissmeyer AM, Krasilnikoff K, Bergh J, Karlsson MO, Lonnebo A and Ahlgren J: A phase I study of chs 828 in patients with solid tumor malignancy. Clin Cancer Res 8(9): 2843-2850, 2002. PMID: 12231525.

10 Olesen UH, Christensen MK, Bjorkling F, Jaattela M, Jensen PB, Sehested M and Nielsen SJ: Anticancer agent chs-828 inhibits cellular synthesis of nad. Biochem Biophys Res Commun 367(4): 799-804, 2008. PMID: 18201551. DOI: 10.1016/j.bbrc.2008.01.019

11 von Heideman A, Berglund A, Larsson R and Nygren P: Safety and efficacy of nad depleting cancer drugs: Results of a phase I clinical trial of chs 828 and overview of published data. Cancer Chemother Pharmacol 65(6): 1165-1172, 2010. PMID: 19789873. DOI: $10.1007 / \mathrm{s} 00280-009-1125-3$

12 Oh A, Ho YC, Zak M, Liu Y, Chen X, Yuen PW, Zheng X, Liu Y, Dragovich PS and Wang W: Structural and biochemical analyses of the catalysis and potency impact of inhibitor phosphoribosylation by human nicotinamide phosphoribosyltransferase. Chembiochem 15(8): 1121-1130, 2014. PMID: 24797455. DOI: $10.1002 /$ cbic.201402023

13 Adams DJ, Ito D, Rees MG, Seashore-Ludlow B, Puyang X, Ramos AH, Cheah JH, Clemons PA, Warmuth M, Zhu P, Shamji 
AF and Schreiber SL: Nampt is the cellular target of stf-31-like small-molecule probes. ACS Chem Biol 9(10): 2247-2254, 2014. PMID: 25058389. DOI: 10.1021/cb500347p

14 Wang W, Elkins K, Oh A, Ho YC, Wu J, Li H, Xiao Y, Kwong M, Coons M, Brillantes B, Cheng E, Crocker L, Dragovich PS, Sampath D, Zheng X, Bair KW, O'Brien T and Belmont LD: Structural basis for resistance to diverse classes of nampt inhibitors. PLoS One 9(10): e109366, 2014. PMID: 25285661. DOI: 10.1371/journal.pone.0109366

15 Watson M, Roulston A, Belec L, Billot X, Marcellus R, Bedard D, Bernier C, Branchaud S, Chan H, Dairi K, Gilbert K, Goulet D, Gratton MO, Isakau H, Jang A, Khadir A, Koch E, Lavoie M, Lawless M, Nguyen M, Paquette D, Turcotte E, Berger A, Mitchell M, Shore GC and Beauparlant P: The small molecule gmx1778 is a potent inhibitor of nad+ biosynthesis: Strategy for enhanced therapy in nicotinic acid phosphoribosyltransferase 1deficient tumors. Mol Cell Biol 29(21): 5872-5888, 2009. PMID: 19703994. DOI: $10.1128 / \mathrm{mcb} .00112-09$

16 Olesen UH, Petersen JG, Garten A, Kiess W, Yoshino J, Imai S, Christensen MK, Fristrup P, Thougaard AV, Bjorkling F, Jensen $\mathrm{PB}$, Nielsen SJ and Sehested M: Target enzyme mutations are the molecular basis for resistance towards pharmacological inhibition of nicotinamide phosphoribosyltransferase. BMC Cancer 10: 677, 2010. PMID: 21144000. DOI: 10.1186/14712407-10-677

17 Ogino Y, Sato A, Uchiumi F and Tanuma SI: Cross resistance to diverse anticancer nicotinamide phosphoribosyltransferase inhibitors induced by fk866 treatment. Oncotarget 9(23): 1645116461, 2018. PMID: 29662658. DOI: 10.18632/oncotarget.24731

18 Ogino Y, Sato A, Uchiumi F and Tanuma SI: Genomic and tumor biological aspects of the anticancer nicotinamide phosphoribosyltransferase inhibitor fk866 in resistant human colorectal cancer cells. Genomics pii:S0888-7543(18)30663-3, 2018. PMID: 30582964. DOI: 10.1016/j.ygeno.2018.12.012

19 Housman G, Byler S, Heerboth S, Lapinska K, Longacre M, Snyder N and Sarkar S: Drug resistance in cancer: An overview. Cancers (Basel) 6(3): 1769-1792, 2014. PMID: 25198391. DOI: $10.3390 /$ cancers6031769

20 Choi $\mathrm{CH}$ : Abc transporters as multidrug resistance mechanisms and the development of chemosensitizers for their reversal. Cancer Cell Int 5: 30, 2005. PMID: 16202168. DOI: $10.1186 / 1475-2867-5-30$
21 Fukuda Y and Schuetz JD: Abc transporters and their role in nucleoside and nucleotide drug resistance. Biochem Pharmacol 83(8): 1073-1083, 2012. PMID: 22285911. DOI: 10.1016/j.bcp. 2011.12.042

22 Choi YH and Yu AM: Abc transporters in multidrug resistance and pharmacokinetics, and strategies for drug development. Curr Pharm Des 20(5): 793-807, 2014. PMID: 23688078. DOI: $10.2174 / 138161282005140214165212$

23 Sato A, Hiramoto A, Uchikubo Y, Miyazaki E, Satake A, Naito T, Hiraoka O, Miyake T, Kim HS and Wataya Y: Gene expression profiles of necrosis and apoptosis induced by 5fluoro-2'-deoxyuridine. Genomics 92(1): 9-17, 2008. PMID: 18572099. DOI: 10.1016/j.ygeno.2008.02.002

24 Sato A, Omi T, Yamamoto A, Satake A, Hiramoto A, Masutani M, Tanuma S, Wataya Y and Kim HS: Microrna-351 regulates two-types of cell death, necrosis and apoptosis, induced by 5fluoro-2'-deoxyuridine. PLoS One 11(4): e0153130, 2016. PMID: 27071035. DOI: 10.1371/journal.pone.0153130

25 Sato A, Satake A, Hiramoto A, Wataya Y and Kim HS: Protein expression profiles of necrosis and apoptosis induced by 5 fluoro-2'-deoxyuridine in mouse cancer cells. J Proteome Res 9(5): 2329-2338, 2010. PMID: 20155980. DOI: 10.1021/ pr9010537

26 O'Connor R: The pharmacology of cancer resistance. Anticancer Res 27(3a): 1267-1272, 2007. PMID: 17593618.

27 Nakanishi T: Drug transporters as targets for cancer chemotherapy. Cancer Genomics Proteomics 4(3): 241-254, 2007. PMID: 17878527.

28 He C, Sun Z, Hoffman RM, Yang Z, Jiang Y, Wang L and Hao $\mathrm{Y}$ : P-glycoprotein overexpression is associated with cisplatin resistance in human osteosarcoma. Anticancer Res 39(4): 17111718, 2019. PMID: 30952710. DOI: 10.21873/anticanres.13277

Received November 1, 2019

Revised November 11, 2019

Accepted November 12, 2019 